\title{
Dynamics of a Host-Parasitoid Model Related to Pennycuick Growth Form
}

\author{
Özlem Ak Gümüşs ${ }^{*}$ and Bekir Sıtkı Bilgi ${ }^{2}$ \\ ${ }^{1}$ Department of Mathematics, Adryaman University, Faculty of Arts and Sciences, 02040, Adiyaman, Turkey \\ ${ }^{2}$ Akkent Anatolian High School, 27070, Sahinbey, Gaziantep, Turkey \\ * Corresponding author
}

\section{Article Info}

Keywords: Discrete-time system, HostParasitoid model, Nicholson Bailey model, Pennycuick form, Stability, Steady state

2010 AMS: 39A30, 91B76, 92B05,

92D25

Received: 8 May 2019

Accepted: 16 September 2019

Available online: 26 December 2019

\begin{abstract}
In this study, the dynamical results of the model by obtaining the steady states existing in the host-parasitoid model were given. Also, some results relating to steady states of the model by depending the parameter made from biological assumptions were obtained.
\end{abstract}

\section{Introduction}

Stability analysis which examines the dynamics of the populations plays an important role in population dynamics. For local asymptotic stability, solutions must approach a steady state under initial conditions close to the steady state. In global asymptotic stability, solutions must approach a steady state under all initial conditions. Since a globally attractive equilibrium point is locally attractive, a globally asymptotically stable steady state is locally asymptotically stable.

It is well known that the Allee effect plays an important role in the stability analysis of the steady states of a population dynamic model (see, for instance, $[4,8,9]$ ). The Allee effect, first introduced by Allee [8], represents a negative density dependence when the population growth rate is reduced at low population size. It may be due to a number of sources including difficulties in finding mates, inbreeding depression, food exploitation, predator avoidance of defense, and social dysfunction at small population sizes. In recent years, the studies on stability of population model with different forms derived from biological facts have attracted many mathematicians [3, 4, 5, 6, 7, 9].

Many ecological models consisting interspecific interactions are generated by differential and difference equations. Especially, the discretetime ecological form with non-overlapping populations are better formulated than continuous-time form. The host-parasitoid models are one of such forms which are studied intensively in the last few decades. One of the earliest applications of discrete-time models including host-parasitoid interaction was obtained by Nicholson and Bailey who applied it to the parasitoid Encarsia formosa and the host Trialeurodes vaporariorum in 1935 [1,2]. Parasitoids are parasites which lay their eggs to host larvae and pupae. Hosts escaping parasitism increase their generation. The searching efficiency of parasitoid increases the number of the parasitized host. The successful parasitized hosts die, but the eggs laid by the parasites can survive for future generations. The general host-parasitoid model proposed by Nicholson-Bailey is presented in the following form

$$
\begin{aligned}
N_{t+1} & =r N_{t} e^{-c P_{t}} \\
P_{t+1} & =e N_{t}\left(1-e^{-c P_{t}}\right) .
\end{aligned}
$$

where $r$ and $e$ are positive parameters. This model assumes as follows:

$N_{t}$ is the density of host species in generation $t$; 
$P_{t}$ is the density of parasitoid species in generation $t$

$r$ is the number of eggs laid by a host that survive through the larvae, pupae and adult stages;

$e$ is the number of eggs laid by parasitoid on a single host that survive through larvae, pupae, adult stages;

$c$ is the searching efficiency.

Also, $e^{-c P_{t}}$ is a fraction of hosts that are not parasitized according to number of encounters under the law of mass action and Poisson distribution.

In this paper, we will investigate the dynamics of a host-parasitoid interaction connected to Pennycuick growth form [10] with different modifications as follows:

$$
\begin{aligned}
H_{t+1} & =\frac{\left(1+a e^{b}\right) H_{t}}{1+a e^{b H_{t}}} e^{-c P_{t}} ; a, b, c>0 \\
P_{t+1} & =H_{t}\left(1-e^{-c P_{t}}\right) .
\end{aligned}
$$

Here, $H_{t}$ is the host population at time $t ; P_{t}$ is the parasitoid population at time $t$. The growth rate of the host population in the absence of the parasitoid, $\frac{\left(1+a e^{b}\right)}{1+a e^{b H_{t}}}$, is associated with the Pennycuick function comes from Pennycuick et al [10].

The aim of this study is to find steady states of the model (1) with and without Allee effect and immigration parameter; and is to investigate the locally asymptotically stability of these steady states.

This paper is regulated as: In Section II, we investigated the steady states of the model (1), and analyzed the locally asymptotically stability of the model (1). In Section III, the steady states of host parasitoid model (1) was examined with immigration parameter. Also, the locally asymptotically stability of this points was investigated. Section IV gives the locally asymptotically stability of the steady states of the host-parasitoid model (1) with Allee effect. Finally, the conclusion is presented.

\section{Steady states of the model (1)}

In this section, we will obtain the steady states of model (1) by using $H_{t}=H_{t+1}=H^{*}$ and $P_{t}=P_{t+1}=P^{*}$ as follows:

$$
\begin{aligned}
H^{*} & =\frac{\left(1+a e^{b}\right) H^{*}}{1+a e^{b H^{*}}} e^{-c P^{*}} ; a, b, c>0 \\
P^{*} & =H^{*}\left(1-e^{-c P^{*}}\right) .
\end{aligned}
$$

Then, we have the following theorem.

Theorem 2.1. The model (1) has the steady states $(0,0),(1,0)$ and $\left(H^{*}, P^{*}\right)$.

Proof. It is clearly seen that $(0,0)$ is a steady state for model (1). Let's take $H^{*} \neq 0$ and $P^{*}=0$. Then we have

$$
H^{*}=\frac{\left(1+a e^{b}\right) H^{*}}{1+a e^{b H^{*}}} .
$$

by from (2). So, we can see that Eq.(3) is provided for $H^{*}=1$. Then $(1,0)$ is steady state of the model (1). Now, we must show that the model (1) has the steady state $\left(H^{*}, P^{*}\right)$ such that $H^{*} \neq 0$ and $P^{*} \neq 0$. If the first equality in (2) is considered, we can write

$$
\begin{aligned}
& H^{*}=\frac{\left(1+a e^{b}\right) H^{*}}{1+a e^{b H^{*}}} e^{-c P^{*}} \\
& \Rightarrow e^{-c P^{*}}=\frac{1+a e^{b H^{*}}}{1+a e^{b}} \\
& \Rightarrow P^{*}=\frac{-1}{c} \ln \frac{1+a e^{b H^{*}}}{1+a e^{b}} .
\end{aligned}
$$

If the following inequality is provided

$$
0<\frac{1+a e^{b H^{*}}}{1+a e^{b}}<1
$$

then $P^{*}>0$ in Eq.(5). We obtain

$$
0<H^{*}<1
$$

by from inequality (6). If we combine Eq.(4) with the second equation of (2), then we get

$$
P^{*}=H^{*}\left(1-\frac{1+a e^{b H^{*}}}{1+a e^{b}}\right)
$$

If $P^{*}$ is written in the first equation in (2), then we obtain

$$
1+a e^{b}=\left(1+a e^{b H^{*}}\right) e^{c H^{*}\left(1-\frac{1+a e^{b H^{*}}}{1+a e^{b}}\right)} .
$$


Let's write the following function by using the right side of (8) such that $H^{*}=x$

$$
f(x)=\left(1+a e^{b x}\right) e^{c x\left(1-\frac{1+a e^{b x}}{1+a e^{b}}\right)} .
$$

Since $(1,0)$ is a steady state of the model (1), we can easily see that $x=1$ is a solution of the Eq.(8). By considering (7), let's investigate some other points providing Eq.(8) apart from $x=1$. In this way, if the derivation of the function $f(x)$ is calculated, we get

$$
f^{\prime}(x)=e^{c x\left(1-\frac{1+a e^{b x}}{1+a e^{b}}\right)}\left[a b e^{b x}+\left(1+a e^{b x}\right)\left(\frac{a c e^{b}}{1+a e^{b}}-\frac{a c e^{b x}+a b c e^{b x} x}{1+a e^{b}}\right)\right] .
$$

From $f^{\prime}(x)=0$,

$$
1=\frac{\left(1+a b e^{b x}\right)}{a b e^{b x}}\left(\frac{a c e^{b x}+a b c e^{b x} x}{1+a e^{b}}-\frac{a c e^{b}}{1+a e^{b}}\right) .
$$

is obtained. Let the function in the right side in Eq.(9) be $F(x)$. Since $F(x)$ is increasing $(F(x)=\infty$ as $x \rightarrow \infty)$, Eq.(9) has an interaction point. Also, since $f(0)=1+a, f^{\prime \prime}(0)=a b+\frac{(1+a) a c e^{b}}{1+a e^{b}}>0$, this critical point is a local maximum for $f(x)$. From this and by considering inequality (7), $f^{\prime}(1)<0$ must be provided. If this inequality is solved, we get the condition $c>1$.
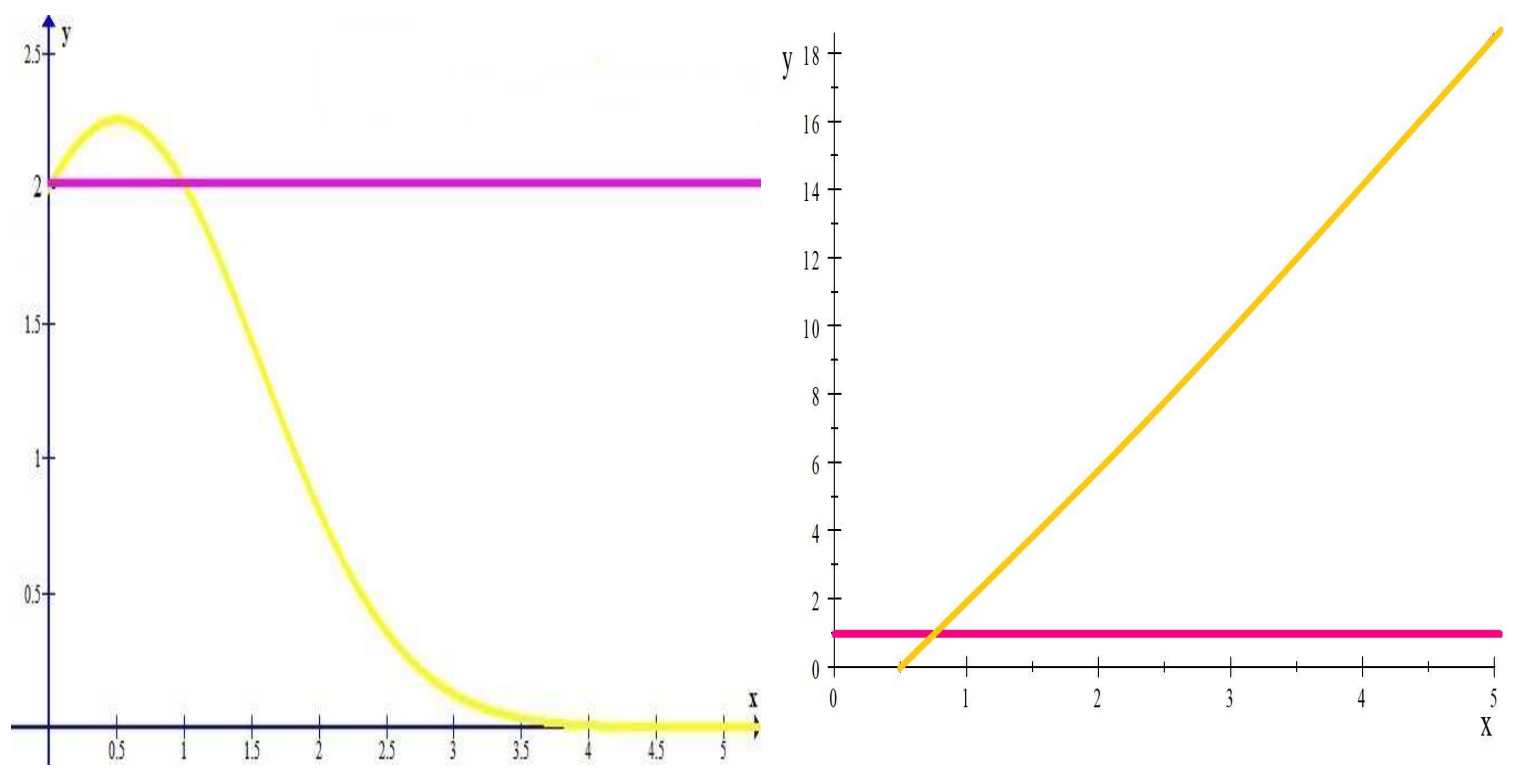

Figure 1: $a \& b$

Figure 2.1- $(a)$ : Graphs of $\left(1+a e^{b}\right)$ and function $f(x)$ where $a=1, b=0.02$ and $c \approx 45.161$.

Figure 2.1-(b): Graphs showing the intersection point $x=0.58208$ where $a=1, b=0.02$ and $c \approx 45.161$.

In Figure 2.1-(a), it is easily seen that the function $f(x)$ has a critical point. If the graphics of the functions Eq. (2.8) are drawn on the same coordinate plane, we observe this interaction point in Fig 2.1-(b).

Corollary 2.2. For the model (1), the following statements hold true:

(a)-If $c \leq 1$, then the model (1) has the steady states $(0,0)$ and $(1,0)$.

(b)-If $c>1$, then the model (1) has the steady states $(0,0),(1,0)$ and $\left(H^{*}, P^{*}\right)$.

\subsection{Stability analysis of model (1)}

In this section, we will investigate the locally asymptotically stability conditions of the steady states of (1).

Theorem 2.3. For the steady states of the model (1), the following statements hold true.

(a)-The steady state $(0,0)$ is not locally asymptotically stable.

(b)-If $2+2 a e^{b}-a b e^{b}>0$ and $c<1$, then the steady state $(1,0)$ is locally asymptotically stable.

(c)-If $c>1$ and under additional conditions, then the steady state $\left(H^{*}, P^{*}\right)$ is locally asymptotically stable.

Proof. (a)-If the model (1) is considered, then we can write

$$
\begin{aligned}
F\left(H_{t}, P_{t}\right) & =\frac{\left(1+a e^{b}\right) H_{t}}{1+a e^{b H_{t}}} e^{-c P_{t}} ; a, b, c>0 \\
G\left(H_{t}, P_{t}\right) & =H_{t}\left(1-e^{-c P_{t}}\right) .
\end{aligned}
$$

Firstly, let' s consider $c \leq 1$. If the Jacobian matrix of model (1) is created in the neighborhood of $(0,0)$, then we have

$$
J_{(0,0)}=\left[\begin{array}{cc}
1+a e^{b} & 0 \\
0 & 0
\end{array}\right] .
$$


The eigenvalues of $J_{(0,0)}$ are $\sigma_{1}=1+a e^{b}$ and $\sigma_{2}=0$. So, $(0,0)$ is locally asymptotically stable if

$$
\left|\sigma_{1}\right|<1 \text { and }\left|\sigma_{2}\right|<1
$$

hold. Since $a e^{b}>0$, one of the inequalities in (10) is not provided. Namely, the steady state $(0,0)$ is not locally asymptotically stable.

(b)-Similarly, if the Jacobian matrix of model (1) is created in the neighborhood of $(1,0)$, then we have

$$
J_{(1,0)}=\left[\begin{array}{cc}
\frac{1+a e^{b}-a b e^{b}}{\left(1+a e^{b}\right)} & -c \\
0 & c
\end{array}\right] .
$$

From this $(1,0)$, is locally asymptotically stable if

$$
|c|<1 \text { and }\left|\frac{1+a e^{b}-a b e^{b}}{\left(1+a e^{b}\right)}\right|<1 \text {. }
$$

hold. We know that $c>0$. If the (11) is solved, we obtain

$$
c<1, a b e^{b}>0 \text { and } 2+2 a e^{b}-a b e^{b}>0 .
$$

Since $a b e^{b}>0$ is always true, $(1,0)$ is locally asymptotically stable under the condition

$$
c<1 \text { and } 2+2 a e^{b}-a b e^{b}>0 .
$$
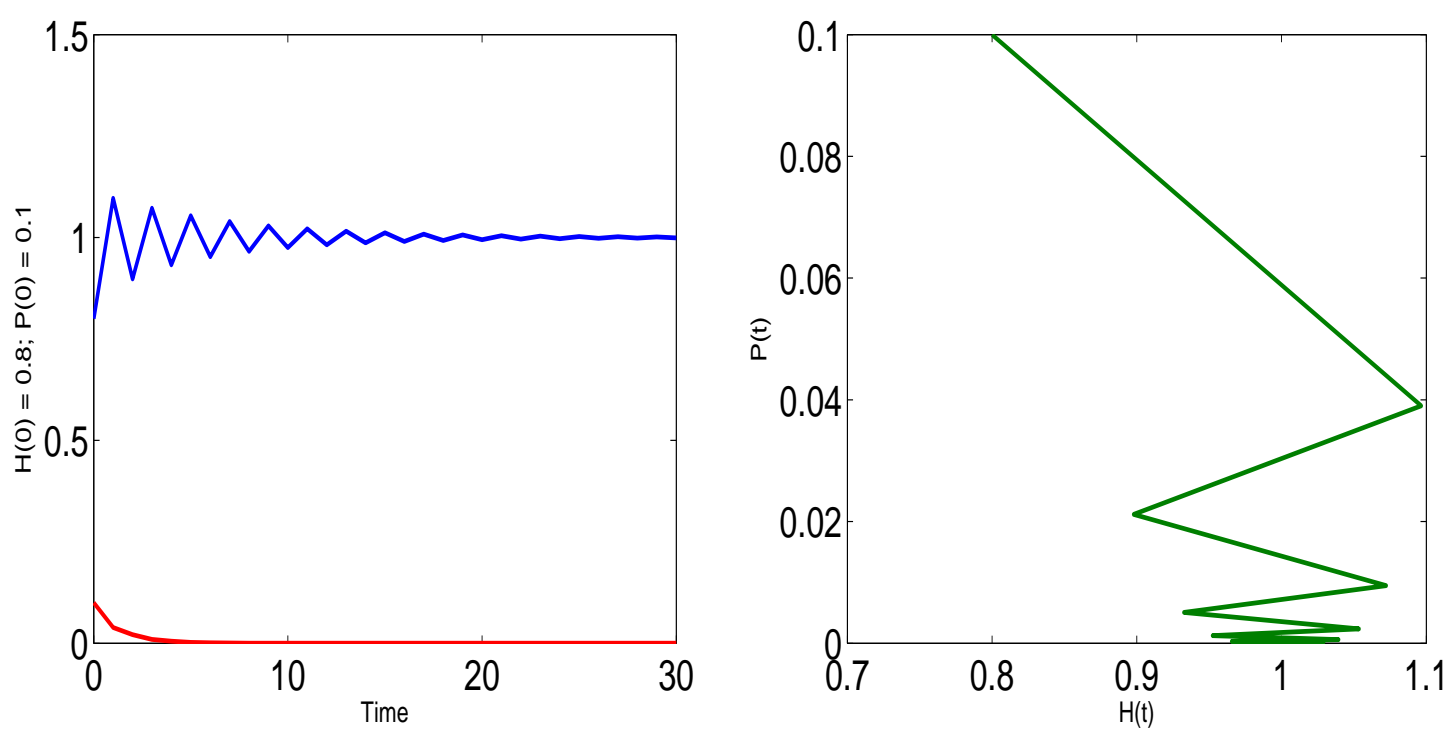

Figure 2: $\mathrm{a} \& \mathrm{~b}$

Figure 2.2-(a): Time series diagram of the model (1) where $a=1.75, b=2$ and $c=0.5$. The initial conditions are $H_{0}=0.8$ and $P_{0}=0.1$. Figure 2.2-(b): Phase diagram of the model (1) where $a=1.75, b=2$ and $c=0.5$. The initial conditions are $H_{0}=0.8$ and $P_{0}=0.1$.

(c)-Finally, let' s consider that $c>1$. The entries of the Jacobian matrix which is evaluated in the neighborhood of $\left(H^{*}, P^{*}\right)$ can be written as follows:

$$
\begin{aligned}
& J_{11}=e^{-c P^{*}}\left(1+a e^{b}\right) \frac{\left[\left(1+a e^{b H^{*}}-a b e^{b H^{*}} H^{*}\right]\right.}{\left(1+a e^{b H^{*}}\right)^{2}} \\
& J_{12}=-c e^{-c P^{*}} \frac{\left(1+a e^{b}\right) H^{*}}{\left(1+a e^{b H^{*}}\right)} \\
& J_{21}=\left(1-e^{-c P^{*}}\right) \\
& J_{22}=c H^{*} e^{-c P^{*}} .
\end{aligned}
$$

From the definition of the determinant and the trace of the matrix $J_{\left(H^{*}, P^{*}\right)}$, we can write

$$
\begin{aligned}
\operatorname{tr} J_{\left(H^{*}, P^{*}\right)} & =e^{-c P^{*}}\left(\frac{\left(1+a e^{b}\right)\left[1+a e^{b H^{*}}-a b e^{b H^{*}} H^{*}\right]}{\left(1+a e^{b H^{*}}\right)^{2}}+c H^{*}\right) \\
\operatorname{det} J_{\left(H^{*}, P^{*}\right)} & =e^{-2 c P^{*}} \frac{\left(1+a e^{b}\right)\left[1+a e^{b H^{*}}-a b e^{b H^{*}} H^{*}\right]}{\left(1+a e^{b H^{*}}\right)^{2}} c H^{*}+c e^{-c P^{*}} \frac{\left(1+a e^{b}\right) H^{*}}{\left(1+a e^{b H^{*}}\right)}\left(1-e^{-c P^{*}}\right) .
\end{aligned}
$$

respectively. If the following inequality (see [2])

$$
|\operatorname{tr} J|<1+\operatorname{det} J<2 .
$$


is provided, then $\left(H^{*}, P^{*}\right)$ is locally asymptotically stable.

By using the inequality (13), we get that $\left(H^{*}, P^{*}\right)$ is locally asymptotically stable if

$$
\begin{aligned}
& e^{-c P^{*}}\left(\frac{\left(1+a e^{b}\right)\left[1+a e^{b H^{*}}-a b e^{b H^{*}} H^{*}\right]}{\left(1+a e^{b H^{*}}\right)^{2}}\right)\left(1-e^{-c P^{*}} c H^{*}\right)+c H^{*} e^{-c P^{*}}-c e^{-c P^{*}} \frac{\left(1+a e^{b}\right) H^{*}}{\left(1+a e^{b H^{*}}\right)}\left(1-e-c P^{*}\right)<1 \\
& e^{-2 c P^{*}} \frac{\left(1+a e^{b}\right)\left[1+a e^{b H^{*}}-a b e^{b H^{*}} H^{*}\right]}{\left(1+a e^{b H^{*}}\right)^{2}} c H^{*}+c e^{-c P^{*}} \frac{\left(1+a e^{b}\right) H^{*}}{\left(1+a e^{\left.b H^{*}\right)}\right.}\left(1-e^{-c P^{*}}\right)<1 \\
& e^{-c P^{*}}\left(\frac{\left(1+a e^{b}\right)\left[1+a e^{b H^{*}}-a b e^{b H^{*}} H^{*}\right]}{\left(1+a e^{b H^{*}}\right)^{2}}\right)\left(1+e^{-c P^{*}} c H^{*}\right)+c H^{*} e^{-c P^{*}}+c e^{-c P^{*}} \frac{\left(1+a e^{b}\right) H^{*}}{\left(1+a e^{b H^{*}}\right)}\left(1-e^{-c P^{*}}\right)>-1
\end{aligned}
$$

such that $c>1$.
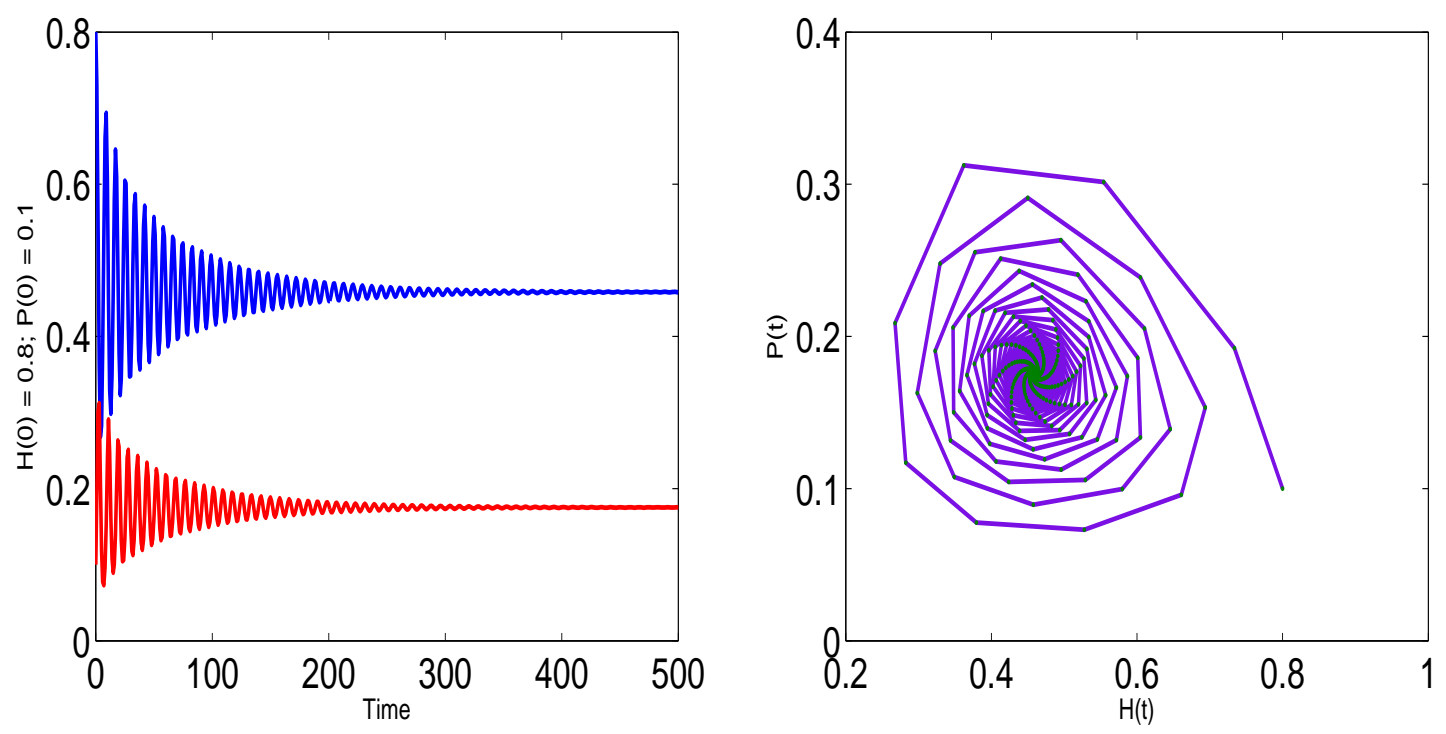

Figure 3: $a \& b$

Figure 2.3-(a): Time series diagram of the model (1) where $a=1, b=1.25$ and $c=2.75$. The initial conditions are $H_{0}=0.8$ and $P_{0}=0.1$. Figure 2.3-(b): Phase Diagram of the model (1) where $a=1, b=1.25$ and $c=2.75$ The initial conditions are $H_{0}=0.8$ and $P_{0}=0.1$.

Corollary 2.4. If the inequality $c \leq 1$ is provided, then the model $(1)$ has the steady states $(0,0)$ and $(1,0)$. The steady state $(0,0)$ is always unstable. The steady state $(1,0)$ is only unique locally asymptotically stable point under condition (12).

Corollary 2.5. If the inequality $c>1$ is provided, then the model (1) has the steady states $(0,0),(1,0)$ and $\left(H^{*}, P^{*}\right)$. The steady state $\left(H^{*}, P^{*}\right)$ is only unique locally asymptotically stable point under conditions (14).

\section{Steady states of the model (1) with immigration parameter}

We will investigate the steady states of the model subject to the parameter $\beta$ into host population in the model (1). Then, the general discrete-time host-population model is

$$
\begin{aligned}
H_{t+1} & =\frac{\left(1+a e^{b}\right) H_{t}}{1+a e^{b H_{t}}} e^{-c P_{t}}+\beta ; a, b, c>0 \\
P_{t+1} & =H_{t}\left(1-e^{-c P_{t}}\right) .
\end{aligned}
$$

Here, $\beta \in(1, \infty)$ is a diffusive force which called as immigration ([3, 4]). Now, let's examine the steady states of the model (16). From $H_{t}=H_{t+1}=H_{1}^{*}$ and $P_{t}=P_{t+1}=P_{1}^{*}$, we can write

$$
\begin{aligned}
& H_{1}^{*}=\frac{\left(1+a e^{b}\right) H_{1}^{*}}{1+a e^{b H_{1}^{*}}} e^{-c P_{1}^{*}}+\beta ; a, b, c>0 \\
& P_{1}^{*}=H_{1}^{*}\left(1-e^{-c P_{1}^{*}}\right) .
\end{aligned}
$$

Then, we have the following theorem.

Theorem 3.1. If $0<\frac{\left(1+a e^{b H_{1}^{*}}\right)\left(H_{1}^{*}-\beta\right)}{\left(1+a e^{b}\right) H_{1}^{*}}<1$ is provided, then the model (16) has the steady state $\left(H_{1}^{*}, 0\right)$ and $\left(H_{1}^{*}, P_{1}^{*}\right)$ such that $H_{1}^{*}>\beta$. Otherwise, $\left(H_{1}^{*}, 0\right)$ is unique steady state. 
Proof. It is clearly seen that there is not the steady state $(0,0)$. Since $\beta \in(1, \infty)$; it must be $H_{1}^{*} \neq 0$. Let's take $H_{1}^{*} \neq 0$ and $P_{1}^{*}=0$. Then we can write

$$
\begin{aligned}
& H_{1}^{*}=\frac{\left(1+a e^{b}\right) H_{1}^{*}}{1+a e^{b H_{1}^{*}}}+\beta \\
& \Rightarrow\left(1+a e^{b}\right)=\frac{\left(1+a e^{b H_{1}^{*}}\right)\left(H_{1}^{*}-\beta\right)}{H_{1}^{*}}, \quad H_{1}^{*}>\beta
\end{aligned}
$$

from Eq.(17). Let's define the following the function such that $H_{1}^{*}=x$,

$$
g(x)=\frac{\left(1+a e^{b x}\right)(x-\beta)}{x}, \quad x \neq 0
$$

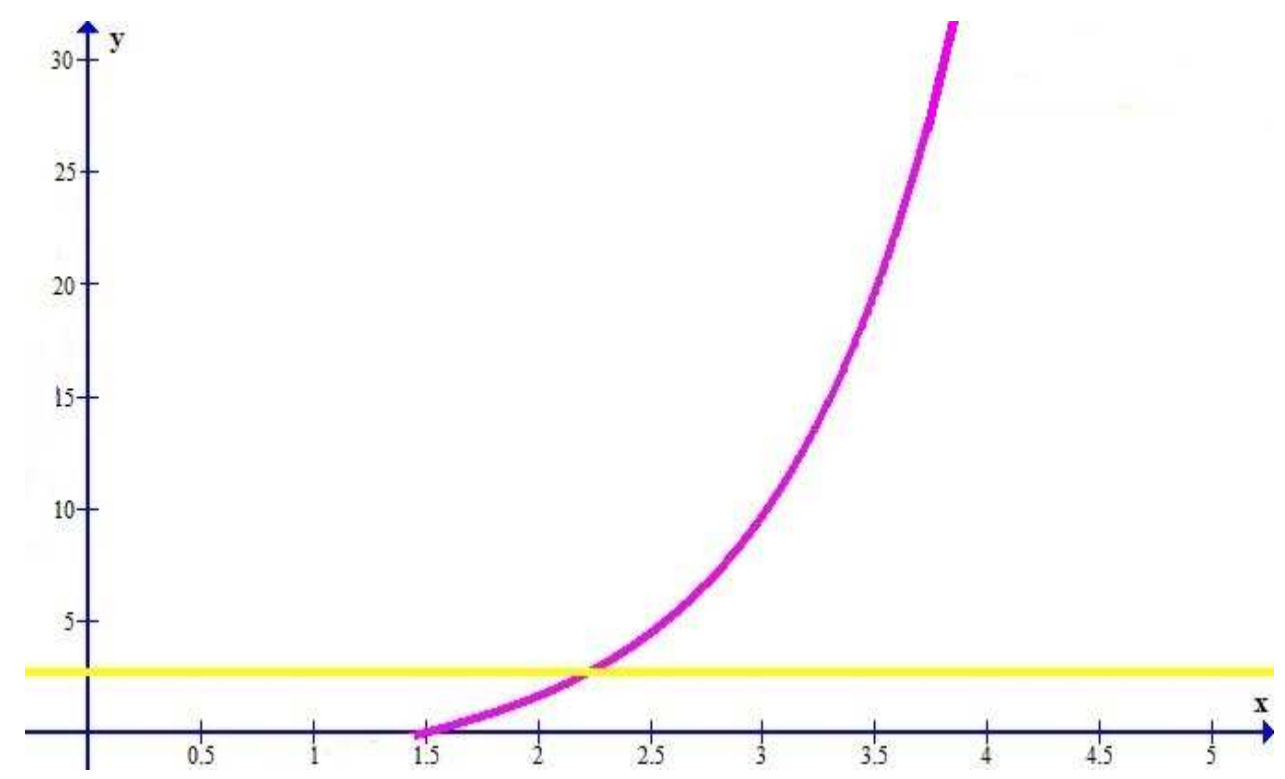

Figure 4: Graphs showing the intersection point

Figure 3.1 Graphs showing the intersection point $H_{1}^{*}$ in (18) where $a=0.5, b=1.2$ and $\beta=1.5$.

From the $g^{\prime}(x)=0$, we have

$$
a b e^{b x}\left(1-\frac{\beta}{x}\right)=-\frac{\beta}{x^{2}}\left(1+a e^{b x}\right)
$$

Eq.(20) has not an interaction point. Also, we can seen that $g(x)=\infty$ as $x \rightarrow \infty$. Since the function $g(x)$ is increasing, Eq.(18) has an interaction point $H_{1}^{*}$.

Now, let's investigate other points $\left(H_{1}^{*}, P_{1}^{*}\right)$ of the model (16) such that $H_{1}^{*} \neq 0$ and $P_{1}^{*} \neq 0$. If the first equality in (16) is considered, we can write

$$
\begin{aligned}
H_{1}^{*} & =\frac{\left(1+a e^{b}\right) H_{1}^{*}}{1+a e^{b H_{1}^{*}}} e^{-c P_{1}^{*}}+\beta \\
\Rightarrow & \left(1+a e^{b H_{1}^{*}}\right)\left(H_{1}^{*}-\beta\right)=\left(1+a e^{b}\right) H_{1}^{*} e^{-c P_{1}^{*}} \\
e^{-c P_{1}^{*}} & =\frac{\left(1+a e^{b H_{1}^{*}}\right)\left(H_{1}^{*}-\beta\right)}{\left(1+a e^{b}\right) H_{1}^{*}} \\
\Rightarrow P_{1}^{*} & =-\frac{1}{c} \ln \frac{\left(1+a e^{b H_{1}^{*}}\right)\left(H_{1}^{*}-\beta\right)}{\left(1+a e^{b}\right) H_{1}^{*}}
\end{aligned}
$$

If the following inequality is provided

$$
0<\frac{\left(1+a e^{b H_{1}^{*}}\right)\left(H_{1}^{*}-\beta\right)}{\left(1+a e^{b}\right) H_{1}^{*}}<1
$$

then $P_{1}^{*}>0$ in (22). Also, we have

$$
\beta<H_{1}^{*} \text { and }\left(1+a e^{b H_{1}^{*}}\right)\left(1-\frac{\beta}{H_{1}^{*}}\right)<1+a e^{b}
$$


from inequality (23). If we combine (21) with the second equation of (16), then we obtain

$$
P_{1}^{*}=H_{1}^{*}\left(1-\frac{\left(1+a e^{b H_{1}^{*}}\right)\left(H_{1}^{*}-\beta\right)}{\left(1+a e^{b}\right) H_{1}^{*}}\right) .
$$

If $P_{1}^{*}$ is written in the first equation in the model (16), then we obtain

$$
\begin{aligned}
& H_{1}^{*}=\frac{\left(1+a e^{b}\right) H_{1}^{*}}{1+a e^{b H_{1}^{*}}} e^{-c H_{1}^{*}\left(1-\frac{\left(1+a e^{b H_{1}^{*}}\right)\left(H_{1}^{*}-\beta\right)}{\left(1+a e^{b}\right) H_{1}^{*}}\right)}+\beta \\
& \Rightarrow 1+a e^{b}=\left(1+a e^{b H_{1}^{*}}\right)\left(1-\frac{\beta}{H_{1}^{*}}\right) e^{c H_{1}^{*}\left(1-\frac{\left(1+a e^{b H_{1}^{*}}\right)\left(H_{1}^{*}-\beta\right)}{\left(1+a e^{b}\right) H_{1}^{*}}\right)}
\end{aligned}
$$

If the previous similar operations are done, it is seen that Eq.(24) has an interaction point. Let's write the following function by using the right side (24)

$$
h(x)=\left(1+a e^{b x}\right)\left(1-\frac{\beta}{x}\right) e^{c x\left(1-\frac{\left(1+a e^{b x}\right)(x-\beta)}{\left(1+a e^{b}\right) x}\right)}
$$

for $H_{1}^{*}=x$. Here, $h(x) \rightarrow 0$ as $x \rightarrow \infty$.

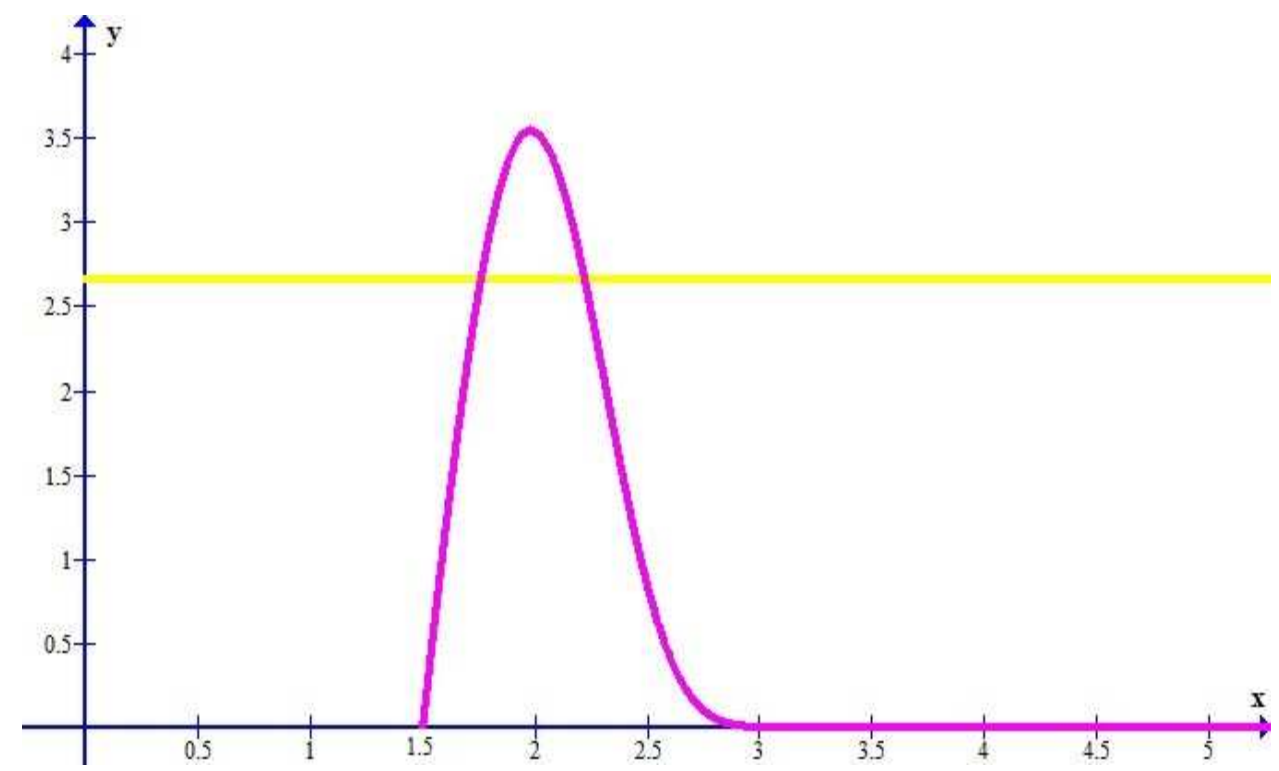

Figure 5: Graphs of $\left(1+a e^{b}\right)$ and function $h(x)$

Figure 3.2 Graphs of $\left(1+a e^{b}\right)$ and function $h(x)$ where $a=0.5, b=1.2, c=1$ and $\beta=1.5$.

\subsection{Stability analysis of model (1) with immigration parameter}

In this section, we will investigate the locally asymptotically stability conditions of steady states of (16). If the model (16) is considered, we can write

$$
\begin{aligned}
F\left(H_{t}, P_{t}\right) & =\frac{\left(1+a e^{b}\right) H_{t}}{1+a e^{b H_{t}}} e^{-c P_{t}}+\beta ; a, b, c>0 \\
G\left(H_{t}, P_{t}\right) & =H_{t}\left(1-e^{-c P_{t}}\right) .
\end{aligned}
$$

Then, we have the following theorem.

Theorem 3.2. For the steady states of the model (16), the following statements hold true:

(a)-Assume that the inequality (23) is not provided. The steady state $\left(H_{1}^{*}, 0\right)$ of the model (16) has the locally asymptotically stable if the conditions (14) are provided for $\left(H_{1}^{*}, 0\right)$.

(b)-Assume that the inequality $(23)$ is provided and $\left(H_{1}^{*}, 0\right)$ unstable. The steady state $\left(H_{1}^{*}, P_{1}^{*}\right)$ of the model $(16)$ has the locally asymptotically stable if the conditions (14) are provided for $\left(H_{1}^{*}, P_{1}^{*}\right)$.

Proof. (a)-On the assumption, $\left(H_{1}^{*}, 0\right)$ is unique steady state of the model (16). If the Jacobian matrix, evaluated in the neighborhood of $\left(H_{1}^{*}, 0\right)$, is written, we get

$$
J_{\left(H_{1}^{*}, 0\right)}=\left[\begin{array}{cc}
\frac{\left(1+a e^{b}\right)\left[\left(1+a e^{b H_{1}^{*}}\right)-a b e^{b H_{1}^{*}} H_{1}^{*}\right]}{\left(1+a e^{b H_{1}^{*}}\right)^{2}} & -c \frac{\left(1+a e^{b}\right) H_{1}^{*}}{1+a e_{1}^{*}} \\
0 & c H_{1}^{*}
\end{array}\right] .
$$


The eigenvalues of $J_{\left(H_{1}^{*}, 0\right)}$ are $\lambda_{1}=\frac{\left(1+a e^{b}\right)\left[\left(1+a e^{b H_{1}^{*}}\right)-a b e^{b H_{1}^{*}} H_{1}^{*}\right]}{\left(1+a e^{b H_{1}^{*}}\right)^{2}}$ and $\lambda_{2}=c H_{1}^{*}$. Consequently, $\left(H_{1}^{*}, 0\right)$ is locally asymptotically stable if

$$
\left|\frac{\left(1+a e^{b}\right)\left[\left(1+a e^{b H_{1}^{*}}\right)-a e^{b H_{1}^{*}} H_{1}^{*}\right]}{\left(1+a e^{b H_{1}^{*}}\right)^{2}}\right|<1 \text { and }\left|c H_{1}^{*}\right|<1 .
$$

We know that $H_{1}^{*}>1$ from Theorem 5 and $c>0$. If the last inequalities are clearly written, we have the following inequalities

$$
\begin{aligned}
& \left(1+a e^{b H_{1}^{*}}\right)^{2}-\left(1+a e^{b}\right)\left[\left(1+a e^{b H_{1}^{*}}\right)-a e^{b H_{1}^{*}} H_{1}^{*}\right]>0 \\
& \left(1+a e^{b H_{1}^{*}}\right)^{2}+\left(1+a e^{b}\right)\left[\left(1+a e^{b H_{1}^{*}}\right)-a e^{b H_{1}^{*}} H_{1}^{*}\right]>0 \\
& c H_{1}^{*}<1 .
\end{aligned}
$$

(b)-On the assumption, we must consider that the conditions (25) are not provided. Then, we can investigate locally asymptotic stability conditions for $\left(H_{1}^{*}, P_{1}^{*}\right)$. The locally asymptotic stability conditions founded for the steady state $\left(H^{*}, P^{*}\right)$ of the model (1) are also applied to stability of the steady state $\left(H_{1}^{*}, P_{1}^{*}\right)$. So, if the conditions $(14)$ are re-written for $\left(H_{1}^{*}, P_{1}^{*}\right)$, then we get as follows:

$$
\begin{aligned}
& e^{-c P_{1}^{*}}\left(\frac{\left(1+a e^{b}\right)\left[1+a e^{b H_{1}^{*}}-a b e^{b H_{1}^{*}} H_{1}^{*}\right]}{\left(1+a e^{b H_{1}^{*}}\right)^{2}}\right)\left(1-e^{-c P_{1}^{*}} c H_{1}^{*}\right)+c H_{1}^{*} e^{-c P_{1}^{*}}-c e^{-c P_{1}^{*}} \frac{\left(1+a e^{b}\right) H_{1}^{*}}{\left(1+a e^{b H_{1}^{*}}\right)}\left(1-e^{-c P_{1}^{*}}\right)<1 \\
& e^{-2 c P_{1}^{*}} \frac{\left(1+a e^{b}\right)\left[1+a e^{b H_{1}^{*}}-a b e^{b H_{1}^{*}} H_{1}^{*}\right]}{\left(1+a e^{b H_{1}^{*}}\right)^{2}} c H_{1}^{*}+c e^{-c P_{1}^{*}} \frac{\left(1+a e^{b}\right) H_{1}^{*}}{\left(1+a e^{b H_{1}^{*}}\right)}\left(1-e^{-c P_{1}^{*}}\right)<1 \\
& e^{-c P_{1}^{*}}\left(\frac{\left(1+a e^{b}\right)\left[1+a e^{b H_{1}^{*}}-a b e^{b H_{1}^{*}} H_{1}^{*}\right]}{\left(1+a e^{b H_{1}^{*}}\right)^{2}}\right)\left(1+e^{-c P_{1}^{*}} c H_{1}^{*}\right)+c H_{1}^{*} e^{-c P_{1}^{*}}+c e^{-c P_{1}^{*}} \frac{\left(1+a e^{b}\right) H_{1}^{*}}{\left(1+a e^{b H_{1}^{*}}\right)}\left(1-e^{-c P_{1}^{*}}\right)>-1
\end{aligned}
$$
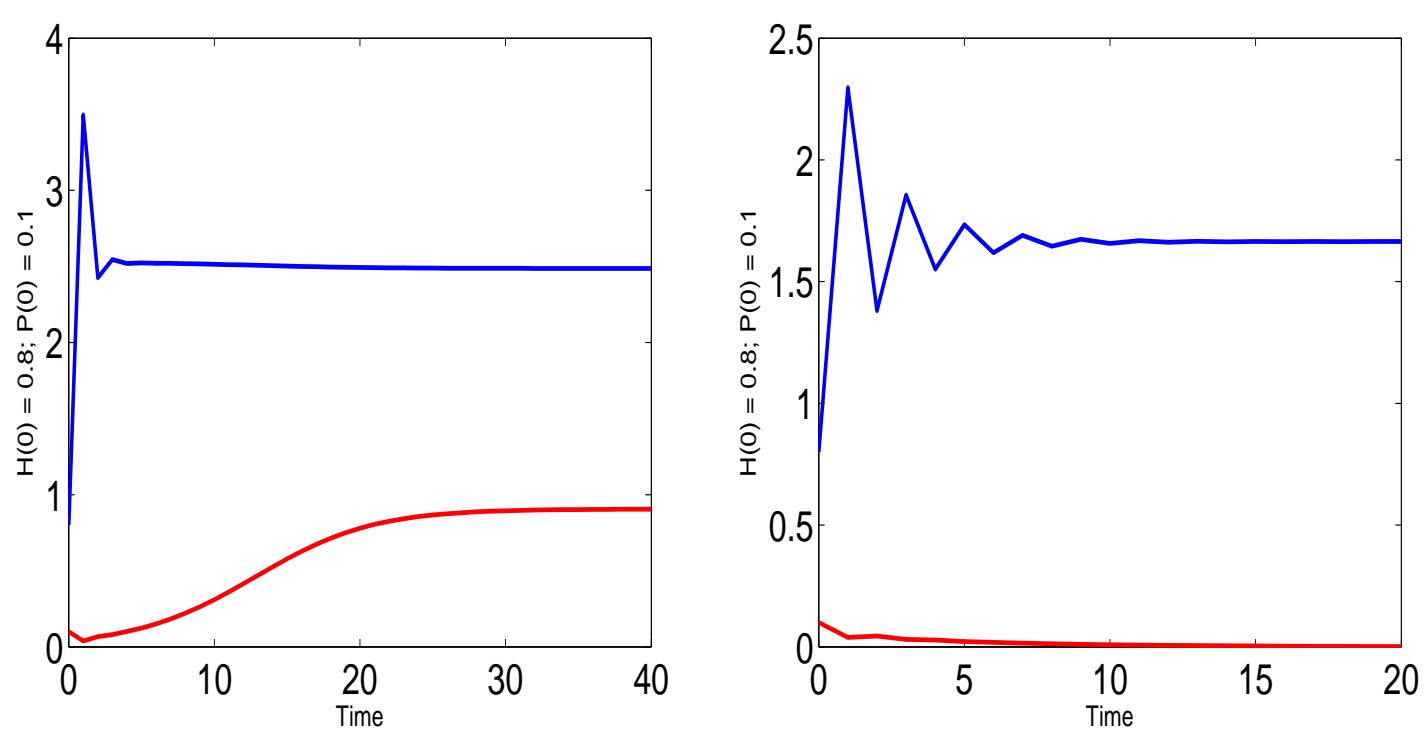

Figure 6: Time series diagram of the model (1)

Figure 3.3. (a): Time series diagram of the model (1) where $a=1, b=2, c=0.5$ and $\beta=2.4$.The initial conditions are $H_{0}=0.8$ and $P_{0}=0.1$.

Figure 3.3. (b): Time series diagram of the model (1) where $a=1, b=2, c=0.5$ and $\beta=1.2$. The initial conditions are $H_{0}=0.8$ and $P_{0}=0.1$.

Corollary 3.3. The steady states $(0,0),(1,0)$ and $\left(H^{*}, P^{*}\right)$ of the model $(1)$ under immigration parameter disappear. The model (1) which subject to immigration parameter appears the steady states $\left(H_{1}^{*}, 0\right)$ and $\left(H_{1}^{*}, P_{1}^{*}\right)$. When inequality $(23)$ is not provided, $\left(H_{1}^{*}, 0\right)$ is unique steady state of the model (16), and it is locally asymptotically stable under (25). Otherwise, the model (16) has steady states $\left(H_{1}^{*}, 0\right)$ and $\left(H_{1}^{*}, P_{1}^{*}\right)$. If $\left(H_{1}^{*}, 0\right)$ unstable, then the steady state $\left(H_{1}^{*}, P_{1}^{*}\right)$ is locally asymptotically stable under $(26)$. 


\section{The stability analysis of model (1) with Allee effect}

We will investigate the steady states of the model by including the Allee effect $\alpha\left(H_{t}\right)$ into host population in the model (1). Then, the general discrete-time host-population model is as follows:

$$
\begin{aligned}
H_{t+1, \alpha} & =\frac{\alpha^{*} H_{t}}{1+a e^{b H_{t}}} e^{-c P_{t}} \alpha\left(H_{t}\right) ; a, b, c>0 \\
P_{t+1} & =H_{t}\left(1-e^{-c P_{t}}\right)
\end{aligned}
$$

where $\alpha^{*}=\left(1+a e^{b}\right) / \alpha$ such that $\alpha: \alpha\left(H_{t}\right)>0$. Therefore, it is clear that the model (1) and the model (28) have the same steady states. The following assumptions on the Allee function $\alpha$ are derived from biological facts:

(i) If there are no partners, there is no reproduction. Mathematically speaking, the Allee function is zero when the population density is zero. (ii) Allee effect increases as density increases. Mathematically speaking, the derivatives of the Allee function are always positive for all positive values.

(iii) Allee effect disappear at high densities. Namely, limit of the Allee function approaches to 1 as the population size increases.

Theorem 4.1. $(0,0)$ is unique locally asymptotically stable steady state of the model (28).

Proof. The entries of the Jacobian matrix associated with the model (28) are given as follows:

$$
\begin{aligned}
& J_{11, \alpha}=\frac{\alpha^{*} e^{-c P_{t}}}{\left(1+a e^{b H_{t}}\right)^{2}}\left(\left[\alpha\left(H_{t}\right)+\alpha^{\prime}\left(H_{t}\right) H_{t}\right]\left(1+a e^{b H_{t}}\right)\right. \\
& \left.-a b e^{b H_{t}} \alpha\left(H_{t}\right) H_{t}\right) \\
& J_{12, \alpha}=-\alpha^{*} c e^{-c P_{t}} \frac{H_{t} \alpha\left(H_{t}\right)}{\left(1+a e^{b H_{t}}\right)} \\
& J_{21, \alpha}=\left(1-e^{-c P_{t}}\right) \\
& J_{22, \alpha}=c H_{t} e^{-c P_{t}} .
\end{aligned}
$$

The Jacobian matrix of the model (28) about the steady state $(1,0)$ is

$$
J_{\alpha(1,0)}=\left[\begin{array}{cc}
\frac{\alpha^{*}}{\left(1+a e^{b}\right)^{2}}\left(\left[\alpha(1)+\alpha^{\prime}(1)\right]\left(1+a e^{b}\right)-a b e^{b} \alpha(1)\right) & \frac{-\alpha^{*} \alpha(1)}{\left(1+a e^{b}\right)} \\
0 & c
\end{array}\right]
$$

Consequently, since $\lambda_{1, \alpha}=\frac{\alpha^{*}}{\left(1+a e^{b}\right)^{2}}\left(\left[\alpha(1)+\alpha^{\prime}(1)\right]\left(1+a e^{b}\right)-a b e^{b} \alpha(1)\right)$ and $\lambda_{2, \alpha}=c$, the steady state $(1,0)$ is locally asymptotically stable if

$$
\left|\frac{\alpha^{*}}{\left(1+a e^{b}\right)^{2}}\left(\left[\alpha(1)+\alpha^{\prime}(1)\right]\left(1+a e^{b}\right)-\alpha^{*} a b e^{b} \alpha(1)\right)\right|<1 \text { and } c<1
$$

Also, by using (29), the entries of the Jacobian matrix of the model (28) of about $\left(H^{*}, P^{*}\right)$ are given as follows:

$$
\begin{aligned}
J_{\alpha, 11} & =\frac{\alpha^{*} e^{-c P^{*}}}{\left(1+a e^{b H^{*}}\right)^{2}}\left(\left[\alpha\left(H^{*}\right)+\alpha^{\prime}\left(H^{*}\right) H^{*}\right]\left(1+a e^{b H^{*}}\right)-a b e^{b H^{*}} \alpha\left(H^{*}\right) H^{*}\right) \\
J_{\alpha, 12} & =-\alpha^{*} c e^{-c P_{t}} \frac{H^{*} \alpha\left(H^{*}\right)}{\left(1+a e^{b H^{*}}\right)} \\
J_{\alpha, 21} & =\left(1-e^{-c P^{*}}\right) \\
J_{\alpha, 22} & =c H^{*} e^{-c P^{*}} .
\end{aligned}
$$

From the definition of the determinant and the trace of the matrix $J_{\left(H^{*}, P^{*}\right)}$, we have

$$
\begin{aligned}
\operatorname{tr} J_{\alpha\left(H^{*}, P^{*}\right)}= & \frac{\alpha^{*} e^{-c P^{*}}}{\left(1+a e^{\left.b H^{*}\right)^{2}}\right.}\left(\left[\alpha\left(H^{*}\right)+\alpha^{\prime}\left(H^{*}\right) H^{*}\right]\left(1+a e^{b H^{*}}\right)\right. \\
& \left.-a b e^{b H^{*}} \alpha\left(H^{*}\right) H^{*}\right)+c H^{*} e^{-c P^{*}} \\
\operatorname{det} J_{\alpha\left(H^{*}, P^{*}\right)}= & \frac{\alpha^{*} e^{-2 c P^{*}} c H^{*}}{\left(1+a e^{b H^{*}}\right)^{2}}\left(\left[\alpha\left(H^{*}\right)+\alpha^{\prime}\left(H^{*}\right) H^{*}\right]\left(1+a e^{b H^{*}}\right)-a b e^{b H^{*}} \alpha\left(H^{*}\right) H^{*}\right) \\
& +\alpha^{*} c e^{-c P_{t}} \frac{H^{*} \alpha\left(H^{*}\right)}{\left(1+a e^{\left.b H^{*}\right)}\right.}\left(1-e^{-c P^{*}}\right) .
\end{aligned}
$$

The (13) yields the following inequality

$$
\begin{array}{c|c}
\left|\begin{array}{c}
\frac{\alpha^{*} e^{-c P^{*}}}{\left(1+a e^{b H^{*}}\right)^{2}}\left(\left[\alpha\left(H^{*}\right)+\alpha^{\prime}\left(H^{*}\right) H^{*}\right]\left(1+a e^{b H^{*}}\right)\right. \\
\left.\left.-a b e^{b H^{*}} \alpha\left(H^{*}\right) H^{*}+c H^{*} e^{-c P^{*}}\right]\right)
\end{array}\right|<1+\frac{\alpha^{*} e^{-2 c P^{*}} c H^{*}}{\left(1+a e^{b H^{*}}\right)^{2}}\left(\left[\alpha\left(H^{*}\right)+\alpha^{\prime}\left(H^{*}\right) H^{*}\right]\left(1+a e^{b H^{*}}\right)-a b e^{b H^{*}} \alpha\left(H^{*}\right) H\right) \\
\alpha^{*} e^{-c P^{*}} \frac{\alpha\left(H^{*}\right) H^{*}}{\left(1+a e^{b H^{*}}\right)}\left(1-e^{-c P^{*}}\right)<2
\end{array}
$$


such that $c>1$. Similarly, the Jacobian matrix of the model $(28)$ about the steady state $(0,0)$ is

$$
J_{\alpha(0,0)}=\left[\begin{array}{ll}
0 & 0 \\
0 & 0
\end{array}\right]
$$

Note that $(0,0)$ is locally asymptotically stable steady state in every situation.

Corollary 4.2. The model (1) with and without Allee effect have the same steady states. As the steady state (1,0) and (H*, $\left.{ }^{*}\right)$ become unstable with Allee effect, the steady state $(0,0)$ becomes locally asymptotically stable. So, $(0,0)$ is unique locally asymptotically stable steady state in the model (1) which subject to Allee effect.

\section{Conclusion}

In this paper, we investigated the steady states of the model (1) with and without immigration parameter and Allee effect. Also, we examined the locally asymptotically stability of steady states of this models. So, we have reached some dynamical consequences which give conditions on stability of the steady states.

\section{References}

[1] A. Nicholson and V. Bailey, The balance of animal population, Proc. Zool. Soc. Lond., 3, (1935).

[2] L.J.S. Allen, An Introduction to Mathematical Biology, Pearson, New Jersey, (2007).

[3] Ö. Ak Gümüş, Dynamical Consequences and Stability Analysis of a New Host-Parasitoid Model, Gen. Math. Notes, 27(1), (2015), 9-15.

[4] Ö. Ak Gümüş, Kangalgil F., Allee effect and stability in a discrete-time host-parasitoid model, J. Adv. Res. Appl. Math., 7(1), (2015), 94-99.

[5] U. Ufuktepe, S. Kapçak, Stability analysis of a host parasite model, Adv. Differ. Equ. doi:10.1186/1687-1847-2013-79.

[6] M. N. Qureshi, A. Q. Khan and Q. Din, Asymptotic behavior of a Nicholson-Bailey model, 62, (2014), doi:10.1186/1687-1847.

[7] A. Q. Khan and M. N. Qureshi, Dynamics of a modified Nicholson-Bailey host-parasitoid model, Adv. Difference Equ., 23, (2015), doi:10.1186/s13662015-0357-2.

[8] W. C. Allee Animal Aggregations: A Study in General Sociology, University of Chicago Press, Chicago (1931).

[9] U. Ufuktepe, S. Kapçak S and O. Akman, Stability analysis of the Beddington model with Allee effect, Appl. Math. Inf. Sci. 9, (2015), 603-608.

[10] C. J. Pennycuick, R. M. Compton and A. Beckingham, A Computer Model for Simulating the Growth of a Population, or of Two Interacting Populations, J. Theoret. Biol., 18, (1968), 316-329. 